\title{
Los desafíos de la población indígena frente a la desigualdad estructural de la educación
}

\section{superior pública guatemalteca}

\section{The challenges of the indigenous population against the structural inequality of Guatemalan public higher education}

\author{
Daniela Josefina Gómez-Willis ${ }^{1}$
}

\section{Resumen}

El presente estudio determina las condiciones de acceso a la educación superior pública en Guatemala, específicamente el análisis se basa en la representatividad de la población indígena en la Universidad de San Carlos de Guatemala, enfatizando los principales obstáculos que limitan su acceso a la educación superior. Para ello se realiza una investigación documental, tomando como referentes los datos oficiales generados por el Departamento de Registro y Estadística de la Universidad de San Carlos de Guatemala, el último Censo Estudiantil Universitario -USAC 2009- y propuestas generadas a partir de las temáticas población indígena y educación superior. Los datos estadísticos permiten visibilizar las desproporciones porcentuales de estudiantes indígenas en los años 2009, 2016 y 2017, en comparación con los estudiantes no indígenas. A partir de los datos generados, surge el análisis y discusión de la necesidad de la existencia de un sistema incluyente, que permita la representación, de la población históricamente desfavorecida e invisibilizada. El objetivo fundamental es dar a conocer los desafíos que enfrenta la población indígena en la búsqueda de la equidad en el acceso a la educación superior pública y el papel de la única universidad pública en Guatemala ante la problemática.

\footnotetext{
1 Daniela Josefina Gómez-Willis. Departamento de Investigación Educativa de la División de Desarrollo Académico y Docencia Universitaria, Universidad de San Carlos de Guatemala. Publicaciones: Deshilando la opresión histórica por el derecho de acceso a la tierra del pueblo q'eqchi' y su resistencia ante nuevos modelos extractivistas. Correo electrónico: danijgw@gmail.com
}

ID: http://orcid.org/0000-0002-9682-6020 
RECIE. Revista Electrónica Científica de Investigación Educativa

Vol. 4, núm. 2, enero-diciembre 2019, pp. 921-930.

\title{
Palabras clave
}

Cultura, derecho a la educación, discriminación racial, desigualdad educativa, desigualdad social.

\begin{abstract}
This study determines the conditions of access to public higher education in Guatemala; specifically, the analysis is based on the representation of the indigenous population at the Universidad de San Carlos de Guatemala, emphasizing the main obstacles that limit their access to higher education. For this, a documentary research was carried out, taking as reference the official data generated by the Department of Registration and Statistics of the Universidad de San Carlos de Guatemala, the last University Student Census -USAC 2009- and proposals generated from the indigenous population issues and higher education. The statistical data allow to visualize the percentage of disproportions of indigenous students in the years 2009, 2016 and 2017, in comparison with non-indigenous students. Based on the data generated, the analysis and discussion leads towards the need for the existence of an inclusive system that allows the representation of the historically disadvantaged and invisivilized population. The main objective of this paper is to raise awareness of the challenges facing the indigenous population in the search for equity in access to public higher education and the role of the only public university in Guatemala in the face of the problem.
\end{abstract}

\section{Keywords}

Culture, right to education, racial discrimination, educational inequality, social inequality.

\section{Contexto: Población indígena y la educación superior pública en Guatemala}

Hablar de equidad permite visualizar una sociedad en donde se tienen las mismas condiciones de acceso a la educación, a la salud, a convivir sin discriminación cultural, entre otros aspectos que hacen posible tener una vida digna. Cómo alcanzar esa equidad social sigue siendo un tema debatible en Guatemala, ya que cuenta con una sociedad multiétnica, pluricultural y multilingüe, con un proceso histórico que ha permitido que aun prevalezca una desigualdad social latente para la población indígena, quienes conforman un $40 \%$ de la población general, de los cuales un $66.8 \%$ 
se encuentra en pobreza extrema, según la Encuesta Nacional de Condiciones de Vida (ENCOVI, 2011).

Las desigualdades para la población indígena también se reflejan en la educación. Según la Primera Encuesta Nacional de Juventud en Guatemala realizada por la Secretaría Ejecutiva de Servicio Cívico (2011), existen variables de mayor peso que afectan el ejercicio del derecho de la educación. La primera es la condición socioeconómica, la segunda variable que afecta las condiciones de desigualdad en la oportunidad de los jóvenes en el acceso a la educación formal, lo constituye la etnicidad, y por último se encuentra el sexo, en donde los hombres generalmente presentan mayores niveles de escolaridad que las mujeres.

"Los indígenas guatemaltecos están insertos en un proceso mediante el cual pretenden eliminar la brecha educativa que les separa de los ladinos, tanto por razones económicas como para poner fin a uno de los factores que determina su subordinación."(Adams, R. y Basto, S; 2003:200). Sin embargo esa brecha persiste, pues en la actualidad los logros son escasos.

Las estadísticas muestran una desproporción étnica en el tema de acceso a la educación. Según el Informe de Desarrollo Humano (2016), el $17.9 \%$ de la población es analfabeta, el 18.9\% no tiene ningún nivel educativo aprobado y el $47.9 \%$ solo tiene estudios a nivel primario. De estos porcentajes el promedio de años de escolaridad para los indígenas es de 4.8, mientras para los no indígenas es de 6.4. El problema se agudiza más si se habla de la oportunidad que tiene la población de llegar a culminar una carrera de nivel medio e ingresar a la educación superior. "En total, solo $1.6 \%$ de hombres y apenas el $0.8 \%$ de mujeres indígenas logran continuar sus estudios superiores, en comparación con el 10.2\% de hombres y 7.3\% de mujeres mestizas" (Kumer, 2013:6).

La desigualdad a la que se enfrenta la población indígena es producto de una herencia colonial, que sigue enraizada en la sociedad contemporánea, en donde la continuidad del fenómeno es observable en las leyes y prácticas institucionales, quienes no asumen como prioritario el tema de desigualdad, naturalizando la desigualdad que se vive en la sociedad (Mato, 2012).

Para el tema de desigualdad en la educación superior, Bourdieu y Paseron (2008) manifiestan que:

En las posibilidades de acceder a la enseñanza superior se lee el resultado de una selección a todo lo largo del recorrido educativo con un rigor muy desigual según el origen social del sujeto. En realidad para la clase más desfavorecida, se trata de una simple y pura eliminación. El hijo de una familia de clase alta tiene ochenta 
veces más chance de entrar a una universidad que el hijo de un asalariado rural.

Así mismo se cuestiona el papel que juega la universidad, ya que "...sin dejar de preferir los intereses de los grupos sociales dominantes, han tratado de dar algunas respuestas (aunque tan solo cosmética) a los interese y grupos sociales dominados" (De Sousa Santos, 2012).

La afirmaciones que generan los distintos pensadores es un punto de partida para dar continuidad a la discusión en torno al tema de desigualdad en el derecho a una educación superior, y el nivel de respuesta ante una realidad que aqueja a la población más vulnerable.

\section{Acceso a la Educación Superior}

El acceso a la educación superior es un tema de debate, más aun si se trata de cuestionar el ingreso a la única universidad pública del país, la Universidad de San Carlos de Guatemala -USAC-, a quien por mandato constitucional "...le corresponde con exclusividad, dirigir, organizar y desarrollar la educación superior del Estado y la educación profesional universitaria estatal, así como la difusión de su cultura en todas sus manifestaciones. Promoverá por todos los medios a su alcance a la investigación en todas las esferas del saber humano y cooperará al estudio y solución de los problemas nacionales" (Leyes y Reglamentos de la USAC, 2009). Así mismo en su Marco Filosófico se expresa que la educación superior debe proyectarse a toda la sociedad, tomando en cuenta el contexto diverso del país (pluricultural, multilingüe y multiétnico).

Ante el compromiso que se le otorga a la Universidad de San Carlos de Guatemala en su carácter de única universidad pública, debe brindar atención a toda la sociedad, por lo tanto en ella tendría que reflejarse la diversidad étnica de la población guatemalteca. Sin embargo el porcentaje de estudiantes indígenas es bajo en comparación con los estudiantes ladinos, siendo uno de los primeros obstáculos para el ingreso a la educación superior el sistema de ingreso, en donde se somete al aspirante a las "pruebas estandarizadas de "conocimientos básicos" asumiendo que todos los estudiantes recibieron los contenidos que están siendo evaluados en el nivel medio y diversificado, y que todos los recibieron con la misma calidad, lo cual está totalmente alejado de la realidad" (Gordillo, 2017: 14).

Al tomar como factor principal los aspectos cognitivos para ingresar a la universidad, la población indígena se encuentra en desventaja con relación a la población ladina quienes tienen acceso a una educación elitista de mayor calidad con respecto a la educación pública que ha ido en 
decadencia, consecuencia del desentendimiento del Estado en cuanto a cobertura y calidad educativa para toda la población guatemalteca. "En estas condiciones de extrema deficiencia, la población indígena sobrelleva una precariedad mayor aun, fruto de su exclusión histórica y de la manera en que se han implementado los programas educativos" (Adams y Basto, 2003:181).

"Si bien el argumento es que no se excluye a nadie por su condición socioeconómica, está claro que en Guatemala la exclusión por conocimientos está directamente relacionada con la condición socioeconómica" (Gordillo, 2017: 14), esto se refleja en el porcentaje de estudiantes que logra matricularse en la universidad, según el último Censo Estudiantil (2009) el 67.92\% de estudiantes proceden de establecimientos privados, el $30.50 \%$ de instituciones educativas públicas, $0.17 \%$ procede de instituciones educativas por cooperativas y el $1.06 \%$ proceden de entidades educativas fuera del país.

Si se analiza el factor étnico de ese mínimo porcentaje de estudiantes provenientes de la educación pública, se puede determinar que la presencia de los estudiantes indígenas en la educación superior aún es débil, lo cual se refleja en la desproporción de los estudiantes inscritos para los años 2009, 2016 y 2017, en donde el porcentaje de estudiantes indígenas es muy bajo en comparación a los estudiantes no indígenas, tal como se muestra en la siguiente tabla:

Tabla 1. Porcentaje de estudiantes inscritos por autodescripción étnica en la Universidad de San Carlos de Guatemala en los años 2009, 2016 y 2017.

\begin{tabular}{|c|c|c|c|c|}
\hline & 2009 & 2016 & 2017 & \multirow{3}{*}{$\begin{array}{c}\text { Estudiantes } \\
\text { no } \\
\text { indígenas }\end{array}$} \\
\hline LADINOS & $72 \%$ & $81 \%$ & $80 \%$ & \\
\hline MESTIZOS & $16 \%$ & $5 \%$ & $8 \%$ & \\
\hline GARIFUNA & $1,1 \%$ & $0,09 \%$ & $0,07 \%$ & \multirow{3}{*}{$\begin{array}{c}\text { Estudiantes } \\
\text { indígenas }\end{array}$} \\
\hline MAYA & $10 \%$ & $13 \%$ & $12 \%$ & \\
\hline XINCA & $0,2 \%$ & $0,10 \%$ & $0,10 \%$ & \\
\hline
\end{tabular}

Fuente: Elaboración propia con base a censo estudiantil USAC 2009 y Departamento de Registro y Estadística de la USAC.

Al realizar la comparación de la presencia de la población indígena entre los años 2009, 2016 y 2017, se puede constatar que no existe una variación significativa de los porcentajes de estudiantes indígenas inscritos en los años analizados. Con ello se puede afirmar que no se han dado cambios 
sustanciales durante ocho años para contrarrestar el problema de desigualdad en el acceso a la educación superior pública.

Los porcentajes que se presentan reflejan un sistema racista y excluyente hacia los pueblos indígenas, porque hasta el momento no se ha presentado la atención que merece un tema tan importante como es la equidad en el acceso a la Universidad de San Carlos de Guatemala, con lo cual se puede determinar que dicha población no es considerada como sujeta de derecho, dando continuidad a la invisibilización de dicha población dentro del sistema educativo.

\section{¿A qué se enfrenta el porcentaje de población indígena que tiene acceso a la educación superior pública?}

El pequeño porcentaje de población indígena que logra ingresar a la educación superior pública, opta por carreras, en su mayoría, del área social humanística, un mínimo porcentaje se encuentra representado en las carreras del área de salud y el área científica tecnológica. Entre algunos factores que influyen en cuanto a la elección de la carrera, se encuentran, según Borrayo (2008), los siguientes:

a) La oferta educacional de su lugar de origen.

b) Situación económica -que sin duda alguna- limita las posibilidades de acceso.

c) El horario disponible de las unidades académicas

d) Ser estudiante trabajador.

e) Por último y en casos excepcionales, los requisitos que deben cumplir cuando se solicita cualquiera de las becas.

Figura No. 1: Porcentaje de estudiantes clasificado por áreas del conocimiento y por autodescripción étnica, según el último censo estudiantil.

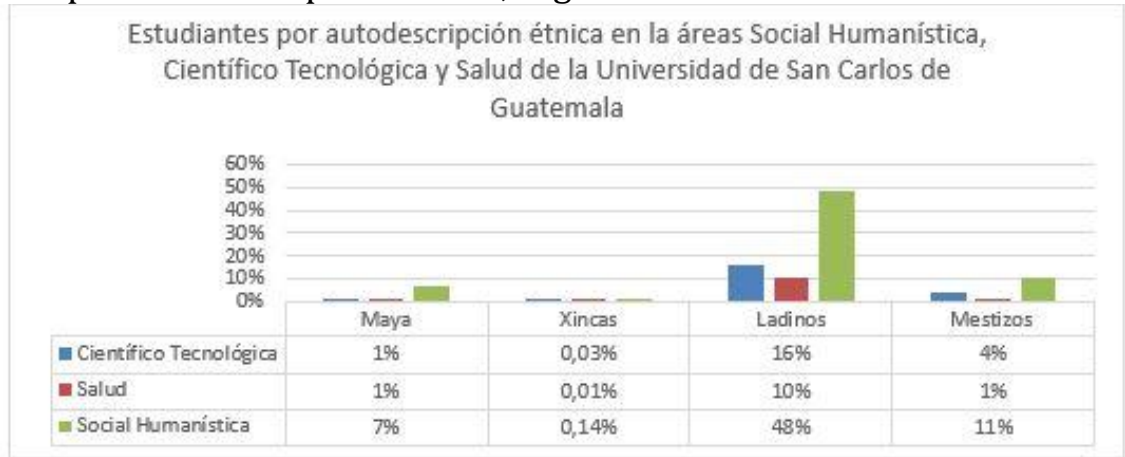

Fuente: elaboración propia en base a III Censo Estudiantil Universitario USAC 2009. 
La Universidad de San Carlos de Guatemala debe reconocer y atender sus múltiples realidades. Si bien es cierto que los estudiantes por su origen étnico y situación socioeconómica desfavorables se encuentran en condiciones de desventaja, esto no significa que su situación de inequidad se siga reproduciendo. La universidad tiene la responsabilidad de compensar $\mathrm{y}$ atender las diferencias con criterios y acciones educativas e institucionales (Gordillo, 2017).

El reto en realidad inicia con buscar los mecanismos que consientan formas equitativas de ingreso a la educación superior pública y cambiar la estructura institucional que permita incluir lo diverso de lo diverso en el ámbito docente, administrativo e investigación como un proyecto educativo que genere nuevos espacios que reflejen la institucionalización genuina de la diversidad.

\section{Las acciones ante la problemática}

En respuesta a todas las responsabilidades que se le asignan a la universidad, en el año 2002 es creado el Plan Estratégico 2022, que tiene como finalidad, por medio de sus objetivos y acciones, brindar atención a las necesidades de la sociedad guatemalteca, para lo cual se crean áreas estratégicas, teniendo entre los principales enfoques el multicultural e intercultural (Plan Estratégico USAC, 2015).

La principal estrategia que busca crear esos espacios de igualdad para la población indígena en la educación superior pública es la Política de Interculturalidad de la Universidad de San Carlos de Guatemala (2017), creada por el Instituto de Estudios Interétnicos en conjunto con la Coordinadora General de Planificación, ambas dependencias de la USAC, que tiene como objetivo primordial:

Construir una universidad incluyente y equitativa hacia la diversidad cultural desde una convivencia intercultural compartida entre sus estudiantes, docentes, profesionales, trabajadores, autoridades y población en general; para garantizar la promoción, reconocimiento y respeto a la diversidad cultural e inclusión de los pueblos indígenas, a través de las funciones de investigación, docencia, extensión y administración.

Son grandes los desafíos que implicaría poner en marcha la Política de Interculturalidad, con lo que se esperaría un ambiente inclusivo, en donde se valore la diversidad, lo cual significaría reconocer, apreciar y respetar las diferencias. La diversidad se debe concebir no como una suma mecánica de 
diferencias, sino como un enfoque multidimensional y multiperspectivista que enfatice su análisis y estudio de las diferencias (Dietz, 2016).

Sobre la importancia de interculturalización de la educación superior Mato (2012) afirma que:

Es un asunto que debería interesar no solo a los pueblos indígenas y afrodescendientes, sino a todos los sectores de las respectivas sociedades nacionales. Porque es condición necesaria para mejorar la calidad de la Educación Superior de los diferentes países, y sus posibilidades de aprovechar mejor las potencialidades de la diversidad de sus poblaciones nacionales y de construir sociedades más equitativas, que sean social y ambientalmente sostenible.

Realizar esa integración de la diversidad étnica, implica valorar y conocer cada uno de los contextos, prácticas culturales y saberes de los distintos pueblos, y sobre todo que ésta población tenga presencia en el sistema educativo.

\section{Reflexiones finales y retos}

El sentido de implementar una política que busque la inclusión de toda la población guatemalteca, sin discriminación alguna, es precisamente porque en la práctica el sistema de ingreso a la única universidad pública es la principal barrera que limita de sobre manera el acceso a la educación superior, contradiciendo las declaraciones y mandatos de hacer de la Universidad de San Carlos de Guatemala una universidad inclusiva.

Ante esta situación, se deben tener cambios más sustanciales, cambios estructurales que permitan reflejar la presencia de la diversidad social guatemalteca con equidad étnica en la educación superior, haciendo valer de esta manera el derecho a la educación. Para lograr la representatividad de la población que históricamente ha sufrido discriminación racial, es necesario implementar "acciones afirmativas" que permitan el empoderamiento y visibilización de los distintos grupos étnicos en los sectores estudiantiles, administrativos, de investigación y docentes de la Universidad de San Carlos de Guatemala.

En la educación superior pública se debe reflejar la población del país por su composición socioeconómica y étnica, por lo tanto, si en los datos oficiales de población se indica que a nivel nacional el $40 \%$ lo conforma la población indígena, en la Universidad de San Carlos de Guatemala tendría que existir ese mismo porcentaje del total de los estudiantes que ingresan cada año, y no la desproporción que actualmente existe entre estudiantes inscritos indígenas y no indígenas. Así mismo es necesario promover que 
las distintas etnias tengan representación en las estructuras administrativas y de gobierno universitario.

Las prácticas de interculturalidad deberían ser fundamentales para la única universidad pública en Guatemala, para la búsqueda de la equidad en el acceso a la educación superior se deben de contar con estrategias, como cursos preuniversitarios (si bien los mismos ya existen, no se le ha dado el enfoque equitativo) que establezcan cuotas de estudiantes indígenas, permitiendo una competencia académica equitativa, en donde la población indígena y no indígena tengan las mismas posibilidades de acceder a una carrera universitaria, disminuyendo los porcentajes de desigualdad en cuanto al acceso a la educación superior.

El reto para la Universidad de San Carlos de Guatemala continúa, la lucha por lograr una equidad en la educación superior sigue siendo un tema prioritario en la sociedad guatemalteca.

\section{Referencias:}

Adams, R. y Bastos, S. (Comp.). (2003) Las relaciones étnicas en Guatemala, 1944-2000. Guatemala: CIRMA.

Borrayo, A. (2008) Experiencias de las mujeres en su acceso a la Universidad de San Carlos de Guatemala, Mayas-Xincas-Garífunas. Guatemala: ARMAR Editores.

Bourdieu, P. y Paseron (2008) Los Herederos: los estudiantes y la cultura. México: Siglo XXI Editores S.A.

De Sousa Santos, B. (2012) De la mano de Alicia: Lo social y lo político en la posmodernidad. Bogotá D.C. Siglo del Hombre Editores.

Dietz, G. (2016) Multiculturalismo, interculturalidad y diversidad en educación. Una aproximación antropológica. México: Fondo de Cultura Económica.

ENCOVI (2011) Caracterización de la República de Guatemala. Guatemala: INE.

Gordillo, E. (2017) Modelos Conceptuales: Estudios sobre el perfil socioeconómico de los estudiantes. Universidad de San Carlos de Guatemala. Guatemala: División de Desarrollo Académico.

Instituto de Estudios Interétnicos y Coordinadora General de Planificación (2017) Propuesta: Política de interculturalidad de la Universidad de San Carlos de Guatemala. Guatemala.

Kummer, S. (2013) Mujeres Indígenas y Educación Superior en Guatemala: Miradas Críticas Sobre su Exclusión. Tesina para especialización en estudios de género. Centro de Investigaciones Interdisciplinarias en 
Ciencias y Humanidades de la Universidad Autónoma de México, Fundación Guatemala, Guatemala.

Mato, D. (2012) Educación Superior y Pueblos indígenas y Afrodescensientes en América Latina: Normas, Políticas y Prácticas. Caracas: IESALCUNESCO.

Programa de Naciones Unidas para el Desarrollo (2016) Más allá del conflicto, luchas por el bienestar. Informe Nacional de Desarrollo Humano 2015-2016. Guatemala.

Secretaría Ejecutiva de Servicio Cívico (2011) Primer encuesta nacional de juventud Guatemala, ENJU 2011. Guatemala.

Universidad de San Carlos de Guatemala (2010) III Censo estudiantil universitario 2009. Primera Edición. Guatemala.

Universidad de San Carlos de Guatemala (2015) Plan Estratégico 2022.

Versión revisada en base al punto cuarto del Acta No. 28-2003. De sesión celebrada por el Consejo Superior Universitario el 26 de noviembre de 2003. Guatemala.

Universidad de San Carlos de Guatemala (2018) Cuadros de estudiantes inscritos en los años 2016 y 2017, por etnia (maya, xinca, ladino, mestizo y garífuna) y sexo. Guatemala: Departamento de Registro y Estadística.

\section{Agradecimientos}

Al Departamento de Investigación Educativa de la DDA-DIGED, de la Universidad de San Carlos de Guatemala. 